PROCEEDINGS OF THE

AMERICAN MATHEMATICAL SOCIETY

Volume 140, Number 4, April 2012, Pages 1413-1422

S 0002-9939(2011)11151-1

Article electronically published on August 16, 2011

\title{
LATTICE STRUCTURES ON SOME BANACH SPACES
}

\author{
MIECZYSŁAW MASTYŁO \\ (Communicated by Marius Junge) \\ To the memory of Nigel Kalton
}

\begin{abstract}
The purpose of this paper is to study Banach lattice constants $d_{n}$ and $e_{n}$ originally introduced by Kalton. We prove an interpolation theorem for positive operators and derive estimates of the lattice constants for Banach lattices generated by positive interpolation functors. In particular, we provide estimates of these constants for Calderón-Loznanovskii spaces. We also find the lattice constants for Marcinkiewicz and Lorentz spaces. As applications, we prove results concerning lattice structures of studied spaces.
\end{abstract}

\section{INTRODUCTION}

The general study of the possible rearrangement-invariant (r.i.) lattice structures on an arbitrary Banach space was initiated in the fundamental work of Johnson, Maurey, Schechtman and Tzafriri [6], which was further developed by Kalton [7]. Kalton's article focussed on the problems of identifying complemented subspaces and the question of uniqueness of lattice structure. In particular, using new ideas he was able to improve and give more definitive results on uniqueness of r.i. structure. It was shown in 7] that Banach lattices not containing uniformly complemented copies of $\ell_{2}^{n}$ play a crucial role. In the study of this type of Banach lattices, Kalton introduced appropriate lattice constants. Following Kalton [7, if $X$ is a Banach lattice we define $d_{n}=d_{n}(X)$ to be the least constant such that for any disjoint $x_{1}, \ldots, x_{n} \in X$

$$
\sum_{k=1}^{n}\left\|x_{k}\right\|_{X} \leq d_{n}\left\|\sum_{k=1}^{n} x_{k}\right\|_{X}
$$

and let $e_{n}=e_{n}(X)$ be the least constant so that for any disjoint $x_{1}, \ldots, x_{n}$,

$$
\left\|\sum_{k=1}^{n} x_{k}\right\|_{X} \leq e_{n} \max _{1 \leq k \leq n}\left\|x_{k}\right\|_{X}
$$

In [7, Theorem 9.4] it was shown that if a Banach lattice $X$ satisfies

$$
\liminf d_{n}(X) / \sqrt{\log n}=0,
$$

Received by the editors August 15, 2010 and, in revised form, January 7, 2011.

2010 Mathematics Subject Classification. Primary 46E30, 46B03, 46M35.

Key words and phrases. Banach lattices, lattice structures, positive operators, positive interpolation functor, Lorentz spaces, Marcinkiewicz spaces, Calderón-Lozanovskii spaces.

This work was supported by the Committee of Scientific Research, Poland, grant No. 201 385034 .

(C)2011 American Mathematical Society Reverts to public domain 28 years from publication 
then $X$ does not contain uniformly complemented $\ell_{2}^{n}$ 's. Moreover, it was also shown in [7, Theorem 9.5 (resp., Theorem 9.6)] that, if $X$ is a separable order-continuous r.i. space on $[0,1]$ such that

$$
\liminf d_{n}(X) / \sqrt{\log n}=0 \quad\left(\text { resp., } \liminf e_{n}(X) / \sqrt{\log n}=0\right)
$$

and $Y$ is a nonatomic Banach lattice isomorphic to a complemented subspace of $X$, then $Y$ is lattice-isomorphic to $X$.

These remarkable results motivate the study of Banach lattice constants defined above. In this paper we provide a general approach based on interpolation of positive operators which allows us to derive estimates of the lattice constants $d_{n}(X)$ and $e_{n}(X)$ for a class of Banach lattices $X$ generated by positive interpolation functors. As an application, we obtain a variant of Kalton's result for r.i. separable CalderónLozanovskii spaces $X=\varphi\left(X_{0}, X_{1}\right)$ generated by couples of Banach lattices $\left(X_{0}, X_{1}\right)$ and positively homogeneous and concave functions $\varphi:[0, \infty) \times[0, \infty) \rightarrow[0, \infty)$. In this special case the condition $\lim \inf d_{n}(X) / \sqrt{\log n}$ may be replaced by

$$
\liminf \frac{\bar{\varphi}\left(d_{n}\left(X_{0}\right), d_{n}\left(X_{1}\right)\right)}{\sqrt{\log n}}=0
$$

where

$$
\bar{\varphi}(s, t)=\sup _{u, v>0} \frac{\varphi(u s, v t)}{\varphi(u, v)}, \quad s, t>0 .
$$

The structure of the exposition is as follows. The abstract approach is described in Section 2, where we prove an interpolation theorem for positive operators and apply it to the study of interpolation estimates of constants $e_{n}$ and $d_{n}$ for CalderónLozanovskii spaces between two given Banach lattices for which we already have estimates of these constants. As an application, we give estimates of these constants for generalized Orlicz spaces. In Section 3, we estimate $e_{n}(M(\psi))$ for the Marcinkiewicz space $M(\psi)$ and $d_{n}(\Lambda(\psi))$ for an order-continuous Lorentz space $\Lambda(\psi)$.

\section{INTERPOLATION ESTIMATES}

Let $(\Omega, \mu):=(\Omega, \Sigma, \mu)$ be a complete $\sigma$-finite measure space and let $X$ be a Banach space. Throughout the paper, $L^{0}(\mu)=L^{0}(\Omega, \mu)$ will denote the space of equivalence classes of strongly measurable $X$-valued functions on $\Omega$, equipped with the topology of convergence in measure. By a Banach lattice on $(\Omega, \mu)$, we shall mean a Banach space $X$ which is a subspace of $L^{0}(\mu)$ such that there exists $u \in X$ with $u>0$, a.e. and such that if $|x| \leq|y|$ a.e., with $y \in X$ and $x \in L^{0}(\mu)$, then we can conclude that $x \in X$ and $\|x\|_{X} \leq\|y\|_{X}$.

A Banach lattice $X$ on $(\Omega, \mu)$ is said to be maximal (resp., order-continuous) if its unit ball $B_{X}=\{x \in X:\|x\| \leq 1\}$ is a closed subset in $L^{0}(\mu)$ (resp., if for every sequence $x_{n} \downarrow 0$, we have $\left\|x_{n}\right\|_{X} \rightarrow 0$ ).

The Köthe dual $X^{\prime}$ of a Banach lattice $X$ on $(\Omega, \mu)$ is defined by

$$
X^{\prime}:=\left\{x \in L^{0}(\mu):\|x\|_{X^{\prime}}=\sup _{\|y\|_{X} \leq 1} \int_{\Omega}|x y| d \mu<\infty\right\} .
$$

Note that $X^{\prime}$ is a Banach lattice under the norm $\|\cdot\|_{X^{\prime}}$ and that the dual space $X^{*}$ is lattice isometric to the Köthe dual space $X^{\prime}$ provided $X$ is order-continuous.

For $f \in L^{0}(\Omega, \mu)$ we define its decreasing rearrangement $f^{*} \in L^{0}([0, \mu(\Omega)))$ by $f^{*}(t)=\inf \{\lambda>0: \mu(|f|>\lambda) \leq t\}$. Let $X$ be a Banach lattice on $([0, a), m)$, 
where $0<a \leq \infty$ and $m$ is the Lebesgue measure. We say that $X$ is a Banach rearrangement invariant space (r.i. space) if $\|f\|_{X}=\left\|f^{*}\right\|_{X}$ for all $f \in X$. If $(\Omega, \mu)$ is a measure space and $X$ is a Banach r.i. space (in which case we shall simply say that $X$ is an r.i. space) on $[0, \mu(\Omega))$, then we define an r.i. space $\widetilde{X}($ on $(\Omega, \mu))$ to be the space of all $f \in L^{0}(\mu)$ such that $f^{*} \in X$ with $\|f\|_{\widetilde{X}}=\left\|f^{*}\right\|_{X}$.

We freely use the standard notions from the interpolation theory from [3]. A mapping $\mathcal{F}$ acting on the class of all couples of Banach lattices, is called a positive interpolation functor if for every couple $\bar{X}=\left(X_{0}, X_{1}\right)$ of Banach lattices, $\mathcal{F}(\bar{X})$ is an intermediate Banach lattice with respect to $\bar{X}$ (i.e., $X_{0} \cap X_{1} \subset \mathcal{F}(\bar{X}) \subset X_{0}+X_{1}$ ), and $T: \mathcal{F}(\bar{X}) \rightarrow \mathcal{F}(\bar{Y})$ for every positive operator $T: \bar{X} \rightarrow \bar{Y}$ between a couple of Banach lattices (meaning that $T: X_{0}+X_{1} \rightarrow Y_{0}+Y_{1}$ is linear and its restrictions $T: X_{j} \rightarrow Y_{j}, j=0,1$ are defined and are positive operators). If in addition there is a constant $C>0$ such that for each $T: \bar{X} \rightarrow \bar{Y}$,

$$
\|T: \mathcal{F}(\bar{X}) \rightarrow \mathcal{F}(\bar{Y})\| \leq C \max \left(\left\|T: X_{0} \rightarrow Y_{0}\right\|,\left\|T: X_{1} \rightarrow Y_{1}\right\|\right),
$$

then $\mathcal{F}$ is called bounded (and exact if $C=1$ ). For a bounded positive interpolation functor $\mathcal{F}$, we define the fundamental function $\psi_{\mathcal{F}}$ of $\mathcal{F}$ by

$$
\psi_{\mathcal{F}}(s, t)=\sup \|T: \mathcal{F}(\bar{X}) \rightarrow \mathcal{F}(\bar{Y})\|
$$

where the supremum is taken over all couples $\bar{X}, \bar{Y}$ of Banach lattices and all positive operators $T: \bar{X} \rightarrow \bar{Y}$ such that $\left\|T: X_{0} \rightarrow Y_{0}\right\| \leq s$ and $\left\|T: X_{1} \rightarrow Y_{1}\right\| \leq t$. From the definition it follows immediately that for any fixed couples $\bar{X}, \bar{Y}$ and $T: \bar{X} \rightarrow \bar{Y}$ we have

$$
\|T: \mathcal{F}(\bar{X}) \rightarrow \mathcal{F}(\bar{Y})\| \leq \psi_{\mathcal{F}}\left(\left\|T: X_{0} \rightarrow Y_{0}\right\|,\left\|T: X_{1} \rightarrow Y_{1}\right\|\right) .
$$

Let $E$ and $F$ be Banach lattices on the measure spaces $\left(\Omega_{1}, \Sigma_{1}, \nu\right)$ and $\left(\Omega_{2}, \Sigma_{2}, \mu\right)$, respectively. We assume (for measurability reasons) that either the measure $\nu$ is discrete or the norm $\|\cdot\|_{F}$ is semi-continuous (i.e., if $0 \leq x_{n} \uparrow x \in E$, then $\left.\left\|x_{n}\right\|_{F} \rightarrow\|x\|_{F}\right)$. A mixed Banach lattice $E[F]$ on $\left(\Omega_{1} \times \Omega_{2}, \nu \times \mu\right)$ is the Banach lattice of all $f \in L^{0}(\nu \times \mu)$ such that $f(\cdot, t) \in F$ for $\mu$-almost all $t \in \Omega_{2}$ and $\|f(\cdot, t)\|_{F} \in E$ with the norm

$$
\|f\|_{E[F]}:=\|\| f(s, t)\left\|_{F, s}\right\|_{E, t} .
$$

For a given Banach lattice on a measure space $(\Omega, \mu)$ and a Banach space $X$, we denote by $E(X)$ the set of all strongly measurable functions $f: \Omega \rightarrow X$ such that $\|f(\cdot)\|_{X} \in E$. This is a Banach space under pointwise operations and a natural norm given by

$$
\|f\|_{E(X)}:=\|\| f(\cdot)\left\|_{X}\right\|_{E} \text {. }
$$

In the study of interpolation Banach spaces the following formula proved by Bukhvalov (see [4, p. 95]) is very useful. It states that for every interpolation functor $\mathcal{F}$ we have

$$
\mathcal{F}\left(E_{0}(X), E_{1}(X)\right)=\mathcal{F}\left(E_{0}, E_{1}\right)(X)
$$

for any couple of Köthe-Bochner spaces $\left(E_{0}(X), E_{1}(X)\right)$. If $\mathcal{F}$ is an exact functor, then the above formula holds with equality of the norms.

We will need a variant of this result for mixed spaces for positive interpolation functors. This result is of independent interest, so here we present a proof. We use the most general version of what is known as the Hahn-Banach extension theorem [1, Theorem 2.1]. 
A map $p: X \rightarrow L^{0}(\mu)$ where $X$ is a (real) vector space is called a sublinear operator if for any $x, y \in X, 0 \leq \lambda \in \mathbb{R}$ we have

$$
\begin{aligned}
p(x+y) & \leq p(x)+p(y), \\
p(\lambda x) & =\lambda p(x) .
\end{aligned}
$$

If $(X, \leq)$ is a lattice, then $p$ is called positive (resp., monotone) whenever $0 \leq x$ (resp., $x \leq y$ ) in $X$ implies $0 \leq p(x)$ (resp., $p(x) \leq p(y)$ ).

Theorem 2.1. Suppose that $\mathcal{F}$ is a positive interpolation functor with the fundamental function $\psi$. Let $\left(X_{0}, X_{1}\right)$ and $\left(Y_{0}, Y_{1}\right)$ be couples of Banach lattices and let $T: X_{0}+X_{1} \rightarrow Y_{0}+Y_{1}$ be a monotone positive sublinear operator such that $\|T x\|_{Y_{j}} \leq M_{j}\|x\|_{X_{j}}$ for every $x \in X_{j}(j=0,1)$. Then $T$ maps $\mathcal{F}\left(X_{0}, X_{1}\right)$ into $\mathcal{F}\left(Y_{0}, Y_{1}\right)$ and we have

$$
\|T x\|_{\mathcal{F}\left(Y_{0}, Y_{1}\right)} \leq \psi\left(M_{0}, M_{1}\right)\|x\|_{\mathcal{F}\left(X_{0}, X_{1}\right)}, \quad x \in \mathcal{F}\left(X_{0}, X_{1}\right) .
$$

Proof. Let $p: X_{0}+X_{1} \rightarrow L^{0}(\mu)$ be defined by $p(x)=T\left(x^{+}\right)$for every $x \in X_{0}+X_{1}$. Fix $x_{0} \in \mathcal{F}\left(X_{0}, X_{1}\right)$ and put $H:=\operatorname{span}\left\{\left|x_{0}\right|\right\}$. Define a positive operator $S_{0}: H \rightarrow$ $L^{0}(\mu)$ by

$$
S_{0}\left(\lambda\left|x_{0}\right|\right)=\lambda T\left(\left|x_{0}\right|\right), \quad \lambda \in \mathbb{R} .
$$

Clearly we have $S_{0}(x) \leq p(x)$ for every $x \in H$. Now, by the Hahn-Banach extension Theorem [1, Theorem 2.1], there exists an extension $S$ of $S_{0}$ to all $X_{0}+X_{1}$ satisfying $S(x) \leq p(x)$ for all $X_{0}+X_{1}$. In particular, if $0 \leq x \in X_{0}+X_{1}$, then

$$
-S(x)=S(-x) \leq p(-x)=T\left((-x)^{+}\right)=T(0)=0
$$

implies that $0 \leq S(x)$ holds. Consequently $S$ is a positive extension of $S_{0}$ to all $X_{0}+X_{1}$. Since $S(x) \leq p(x)=T\left(x^{+}\right)$and $|S(x)| \leq S(|x|)$ for every $x \in X_{0}+X_{1}$, our hypothesis on $T$ yields

$$
\|S x\|_{Y_{j}} \leq\left\|T\left(x^{+}\right)\right\|_{Y_{j}} \leq M_{j}\|x\|_{X_{j}}
$$

for all $x \in X_{j}(j=0,1)$. Combining these remarks with the interpolation property, we obtain $T\left(\left|x_{0}\right|\right)=S\left(\left|x_{0}\right|\right) \in \mathcal{F}\left(X_{0}, X_{1}\right)$ and

$$
\left\|T\left(\left|x_{0}\right|\right)\right\|_{\mathcal{F}\left(Y_{0}, Y_{1}\right)} \leq \psi\left(M_{0}, M_{1}\right)\left\|x_{0}\right\|_{\mathcal{F}\left(X_{0}, X_{1}\right)} .
$$

If, in addition, $T$ is monotone, then the desired inequality follows by $\left|T\left(x_{0}\right)\right| \leq$ $T\left(\left|x_{0}\right|\right)$.

Corollary 2.2. Let $\mathcal{F}$ be a bounded positive interpolation functor. Then we have

$$
\mathcal{F}\left(E_{0}[F], E_{1}[F]\right)=\mathcal{F}\left(E_{0}, E_{1}\right)[F]
$$

for any Banach lattices $E_{0}, E_{1}$ on $\left(\Omega_{1}, \nu\right)$ and $F$ on $\left(\Omega_{2}, \mu\right)$. If in addition $\mathcal{F}$ is an exact functor, then the above formula holds with the equality of the norms.

Proof. Define a monotone positive sublinear operator $S: E_{0}[F]+E_{1}[F] \rightarrow L^{0}(\mu)$ by

$$
S f(\cdot)=\|f(\cdot, t)\|_{F}, \quad f \in E_{0}[F]+E_{1}[F] .
$$

Since $S: E_{j}[F] \rightarrow E_{j}$ is bounded with the norm less than or equal to 1 for $j=0$ and $j=1$, it follows by Theorem 2.1 that

$$
S: \mathcal{F}\left(E_{0}[F], E_{1}[F]\right) \rightarrow \mathcal{F}\left(E_{0}, E_{1}\right)
$$

with $\|S\| \leq 1$. This implies that the inclusion map

$$
\mathcal{F}\left(E_{0}[F], E_{1}[F]\right) \hookrightarrow \mathcal{F}\left(E_{0}, E_{1}\right)[F]
$$


is bounded with the norm less than or equal to 1 . Now fix $f \in \mathcal{F}\left(E_{0}, E_{1}\right)[F]$ and define a positive linear operator $T: E_{0}+E_{1} \rightarrow E_{0}[F]+E_{1}[F]$ by

$$
T x(s, t):=x(t) \frac{|f(s, t)|}{\|f(\cdot, t)\|_{F}}
$$

for every $x \in E_{0}+E_{1}$ and $(s, t) \in \Omega_{1} \times \Omega_{2}$. Clearly $\|T x\|_{E_{j}[F]}=\|x\|_{E_{j}}$ for $j=0,1$ and $T\left(\|f(\cdot, t)\|_{F}\right)=|f|$. This implies by the interpolation property that the inclusion map

$$
\mathcal{F}\left(E_{0}, E_{1}\right)[F] \hookrightarrow \mathcal{F}\left(E_{0}[F], E_{1}[F]\right)
$$

is bounded with the norm less than or equal to 1 , and this completes the proof.

Before showing applications of the above theorem, we state the following result. The proof of the first inequality is an easy observation, and the second one follows from the proof of Proposition 9.2 in [7] presented for abstract separable ordercontinuous Banach lattices.

Proposition 2.3. Let $X$ be an order-continuous Banach lattice on $(\Omega, \mu)$. Then $d_{n}(X)=e_{n}\left(X^{\prime}\right)$ and $d_{n}\left(X^{\prime}\right)=e_{n}(X)$. Moreover, if $x_{1}, \ldots, x_{n}$ are any elements in $X$, then we have

$$
\begin{gathered}
\sum_{k=1}^{n}\left\|x_{k}\right\|_{X} \leq d_{n}(X)\left\|\sum_{k=1}^{n}\left|x_{n}\right|\right\|_{X}, \\
\left\|\max _{1 \leq k \leq n}\left|x_{k}\right|\right\|_{X} \leq e_{n}(X) \max _{1 \leq k \leq n}\left\|x_{k}\right\|_{X} .
\end{gathered}
$$

Now we are ready to show some applications. We first give an illustrative lemma for Banach lattices generated by bounded positive interpolation functors.

Lemma 2.4. Let $\mathcal{F}$ be a bounded positive interpolation functor with fundamental function $\psi$. Then for every couple $\left(X_{0}, X_{1}\right)$ of Banach lattices we have

(i) If $C_{n, 1}:=\left\|i d: \mathcal{F}\left(\ell_{1}^{n}\left(X_{0}\right), \ell_{1}^{n}\left(X_{1}\right)\right) \rightarrow \ell_{1}^{n}\left(\mathcal{F}\left(X_{0}, X_{1}\right)\right)\right\|$ for each $n \in \mathbb{N}$, then

$$
d_{n}\left(\mathcal{F}\left(X_{0}, X_{1}\right)\right) \leq C_{n, 1} \psi\left(d_{n}\left(X_{0}\right), d_{n}\left(X_{1}\right)\right) .
$$

(ii) If $C_{n, \infty}:=\left\|i d: \ell_{\infty}^{n}\left(\mathcal{F}\left(X_{0}, X_{1}\right)\right) \rightarrow \mathcal{F}\left(\ell_{\infty}^{n}\left(X_{0}\right), \ell_{\infty}^{n}\left(X_{1}\right)\right)\right\|$ for each $n \in \mathbb{N}$, then

$$
e_{n}\left(\mathcal{F}\left(X_{0}, X_{1}\right)\right) \leq C_{n, \infty} \psi\left(e_{n}\left(X_{0}\right), e_{n}\left(X_{1}\right)\right) .
$$

Proof. (i). Fix a positive integer $n$. Let $i d$ be a map defined by $i d\left(x_{1}, \ldots, x_{n}\right):=$ $\left(x_{1}, \ldots, x_{n}\right)$ for every $\left(x_{1}, \ldots, x_{n}\right) \in X_{0}\left[\ell_{1}^{n}\right]+X_{1}\left[\ell_{1}^{n}\right]$. Clearly

$$
i d:\left(X_{0}\left[\ell_{1}^{n}\right], X_{1}\left[\ell_{1}^{n}\right]\right) \rightarrow\left(\ell_{1}^{n}\left(X_{0}\right), \ell_{1}^{n}\left(X_{1}\right)\right)
$$

is a positive linear operator. By Proposition 2.3, we have $\left\|i d: X_{j}\left[\ell_{1}^{n}\right] \rightarrow \ell_{1}^{n}\left(X_{j}\right)\right\|=$ $d_{n}\left(X_{j}\right)$ for $j=0,1$. Thus, applying Corollary 2.2 we obtain

$$
i d: \mathcal{F}\left(X_{0}, X_{1}\right)\left[\ell_{1}^{n}\right] \rightarrow \mathcal{F}\left(\ell_{1}^{n}\left(X_{0}\right), \ell_{1}^{n}\left(X_{1}\right)\right)
$$

with

$$
\|i d\| \leq \psi\left(d_{n}\left(X_{0}\right), d_{n}\left(X_{1}\right)\right)
$$

Since $d_{n}\left(\mathcal{F}\left(X_{0}, X_{1}\right)\right)=\left\|i d: \mathcal{F}\left(X_{0}, X_{1}\right)\left[\ell_{1}^{n}\right] \rightarrow \ell_{1}^{n}\left(\mathcal{F}\left(X_{0}, X_{1}\right)\right)\right\|$, the desired estimate follows. The proof of (ii) is similar. 
In what follows the set of all functions $\varphi:[0, \infty) \times[0, \infty) \rightarrow[0, \infty)$ which are nondecreasing in each variable and are positively homogeneous (that is, $\varphi(\lambda s, \lambda t)=$ $\lambda \varphi(s, t)$ for all $\lambda, s, t \geq 0)$ is denoted by $\Phi$. If in addition $\varphi$ is concave, then we write $\varphi \in \mathcal{U}$. For any $\varphi \in \Phi$ we define the functions $\varphi_{*}, \bar{\varphi} \in \Phi$ by $\varphi_{*}(s, t)=1 / \varphi\left(s^{-1}, t^{-1}\right)$ for every $s, t>0, \varphi_{*}(0,0):=0$ and

$$
\bar{\varphi}(s, t)=\sup \left\{\frac{\varphi(u s, v t)}{\varphi(u, v)}: u, v>0\right\}, \quad s, t \geq 0 .
$$

Recall that if $\bar{X}=\left(X_{0}, X_{1}\right)$ is a couple of Banach lattices on $(\Omega, \mu)$ and $\varphi \in \mathcal{U}$, then the Calderón-Lozanovskii space $\varphi(\bar{X})=\varphi\left(X_{0}, X_{1}\right)$ consists of all $x \in L^{0}(\mu)$ such that $|x| \leq \lambda \varphi\left(\left|x_{0}\right|,\left|x_{1}\right|\right) \mu$-a.e. on $\Omega$ for some $x_{j} \in E_{j}$ with $\left\|x_{j}\right\|_{X_{j}} \leq 1$, $j=0,1$. The space $\varphi(\bar{X})$ is a Banach lattice equipped with the norm (see [11, [12])

$$
\|x\|_{\varphi(\bar{X})}:=\inf \left\{\lambda>0:|x| \leq \lambda \varphi\left(\left|x_{0}\right|,\left|x_{1}\right|\right),\left\|x_{0}\right\|_{X_{0}} \leq 1,\left\|x_{1}\right\|_{X_{1}} \leq 1\right\} .
$$

In the case of the power function $\varphi(s, t)=s^{1-\theta} t^{\theta}$ with $0<\theta<1, \varphi(\bar{X})$ is the well-known Calderón space denoted by $X_{0}^{1-\theta} X_{1}^{\theta}$ (see [5]).

Notice that a Calderón-Lozanovskii space can be defined as the space of all functions $x \in L^{0}(\mu)$ such that $|x|=\varphi\left(\left|x_{0}\right|,\left|x_{1}\right|\right)$ for some $x_{0} \in X_{0}, x_{1} \in X_{1}$ with the norm

$$
\|x\|_{\varphi}=\inf \max \left\{\left\|x_{0}\right\|_{X_{0}},\left\|x_{1}\right\|_{X_{1}}\right\}
$$

where the infimum is taken over all $x_{0}, x_{1}$ for which $|x|=\varphi\left(\left|x_{0}\right|,\left|x_{1}\right|\right)$.

Theorem 2.5. Let $\left(X_{0}, X_{1}\right)$ be a couple of Banach lattices and $\varphi \in \mathcal{U}$. Then we have

$$
d_{n}\left(\varphi\left(X_{0}, X_{1}\right)\right) \leq 2 \bar{\varphi}\left(d_{n}\left(X_{0}\right), d_{n}\left(X_{1}\right)\right)
$$

and

$$
e_{n}\left(\varphi\left(X_{0}, X_{1}\right)\right) \leq \bar{\varphi}\left(e_{n}\left(X_{0}\right), e_{n}\left(X_{1}\right)\right) .
$$

Proof. We apply Lemma 2.4. Since $\mathcal{F}(\cdot)=\varphi(\cdot)$ is a positive interpolation functor with the fundamental function $\psi \leq \bar{\varphi}$ (see [15]), it is sufficient to show that for each $n$,

$$
\begin{gathered}
C_{n, 1}=\left\|i d: \varphi\left(\ell_{1}^{n}\left(X_{0}\right), \ell_{1}^{n}\left(X_{1}\right)\right) \rightarrow \ell_{1}^{n}\left(\varphi\left(X_{0}, X_{1}\right)\right)\right\| \leq 2, \\
C_{n, \infty}=\left\|i d: \ell_{\infty}^{n}\left(\varphi\left(X_{0}, X_{1}\right)\right) \rightarrow \varphi\left(\ell_{\infty}^{n}\left(X_{0}\right), \ell_{\infty}^{n}\left(X_{1}\right)\right)\right\| \leq 1 .
\end{gathered}
$$

Notice that for every $\left(x_{j}\right)_{j=1}^{n}:=\left(\varphi\left(\left|u_{j}\right|,\left|v_{j}\right|\right)\right)_{j=1}^{n} \in \varphi\left(\ell_{1}^{n}\left(X_{0}\right), \ell_{1}^{n}\left(X_{1}\right)\right)$, we have

$$
\begin{aligned}
\sum_{j=1}^{n}\left\|x_{j}\right\|_{\varphi(\bar{X})} & \leq \sum_{j=1}^{n} \max \left\{\left\|u_{j}\right\|_{X_{0}},\left\|v_{j}\right\|_{X_{1}}\right\} \\
& \leq 2 \max \left\{\left\|\left(u_{j}\right)_{j=1}^{n}\right\|_{\ell_{1}^{n}\left(X_{0}\right)},\left\|\left(v_{j}\right)_{j=1}^{n}\right\|_{\ell_{1}^{n}\left(X_{1}\right)}\right\},
\end{aligned}
$$

whence the first estimate shown above follows. The estimate of $C_{n, \infty}$ is also obvious.

Theorem 2.6. Let $\varphi \in \mathcal{U}$ satisfy $\bar{\varphi}(s, 1) \rightarrow 0$ as $s \rightarrow 0$ and let $\left(X_{0}, X_{1}\right)$ be a couple of r.i. spaces on $[0,1]$ such that $X_{0}$ is a separable space and satisfies

$$
\liminf \frac{\bar{\varphi}\left(d_{n}\left(X_{0}\right), d_{n}\left(X_{1}\right)\right)}{\sqrt{\log n}}=0 .
$$


If $Y$ is a nonatomic Banach lattice isomorphic to a complemented subspace of $\varphi\left(X_{0}, X_{1}\right)$, then $Y$ is lattice-isomorphic to $\varphi\left(X_{0}, X_{1}\right)$.

Proof. Clearly $\varphi\left(X_{0}, X_{1}\right)$ is an r.i. space on $[0,1]$. Our hypotheses on $\varphi$ and $X_{0}$ imply that $\varphi\left(X_{0}, X_{1}\right)$ is separable (see [14); moreover, Theorem 2.5] implies that

$$
\liminf \frac{d_{n}\left(\varphi\left(X_{0}, X_{1}\right)\right)}{\sqrt{\log n}}=0 .
$$

Thus the statement follows by [7, Theorem 9.5].

We show applications to generalized Orlicz spaces. Let $\varphi:[0, \infty) \rightarrow[0, \infty)$ be an Orlicz function (i.e., $\varphi(s)=0, \varphi$ is nondecreasing convex and left continuous). Put $\rho(s, t):=t \varphi^{-1}(s / t), t>0$ and 0 if $t=0$, where $\varphi^{-1}$ is the right continuous inverse of $\varphi$. Then it is easy to check that for any Banach lattice $X$ on $(\Omega, \mu)$, we have

$$
\rho\left(X, L_{\infty}\right)=X_{\varphi}:=\left\{x \in L^{0}(\mu): \varphi(|x| / \lambda) \in X \text { for some } \lambda>0\right\}
$$

and

$$
\|x\|_{\rho}=\|x\|_{X_{\varphi}}:=\inf \left\{\lambda>0:\|\varphi(|x| / \lambda)\|_{X} \leq 1\right\} .
$$

In what follows if $f:(0, \infty) \rightarrow(0, \infty)$, we define $\bar{f}(t):=\sup \{f(s t) / f(s): s>0\}$ for all $t>0$. Since $d_{n}\left(L_{\infty}\right)=n$ and $e_{n}\left(L_{\infty}\right)=1$, the following corollary is an immediate consequence of Theorem 2.5.

Corollary 2.7. Let $X$ be a Banach lattice on $(\Omega, \mu)$ and $\varphi$ be an Orlicz function. Then for all $n \in \mathbb{N}$ we have

$$
d_{n}\left(X_{\varphi}\right) \leq 2 n \overline{\varphi^{-1}}\left(\frac{d_{n}(X)}{n}\right)
$$

and

$$
e_{n}\left(X_{\varphi}\right) \leq \overline{\varphi^{-1}}\left(e_{n}(X)\right) .
$$

We now give asymptotic estimates of the constants $d_{n}$ and $e_{n}$ for Orlicz spaces over infinite nonatomic measure spaces.

Theorem 2.8. Let $L_{\varphi}$ be an Orlicz space on a nonatomic measure space $(\Omega, \mu)$ with $\mu(\Omega)=\infty$. Then for each positive integer $n$ we have

$$
n \overline{\varphi^{-1}}(1 / n) \leq d_{n}\left(L_{\varphi}\right) \leq 2 n \overline{\varphi^{-1}}(1 / n)
$$

and

$$
e_{n}\left(L_{\varphi}\right)=\overline{\varphi^{-1}}(n)
$$

Proof. Fix $t>0$ and $n \geq 2$. Since $\mu$ is nonatomic measure, we may find a measurable subset $A \subset \Omega$ and disjoint measurable subsets $A_{1}, \ldots, A_{n}$ of $A$ with $\mu(A)=t$, $A=\bigcup_{k=1}^{n} A_{k}$ so that $\mu\left(A_{k}\right)=t / n$ for each $k=1, \ldots, n$. Then for $f_{k}=\chi_{A_{k}}$ with $k=1, \ldots, n$ we have

$$
\begin{gathered}
\frac{n}{\varphi^{-1}(n / t)}=\sum_{k=1}^{n}\left\|f_{k}\right\|_{L_{\varphi}} \leq d_{n}\left(L_{\varphi}\right)\left\|\sum_{k=1}^{n} f_{k}\right\|_{L_{\varphi}}=\frac{d_{n}\left(L_{\varphi}\right)}{\varphi^{-1}(1 / t)} \\
\frac{1}{\varphi^{-1}(1 / t)}=\left\|\sum_{k=1}^{n} f_{k}\right\|_{L_{\varphi}} \leq e_{n}\left(L_{\varphi}\right) \max _{1 \leq k \leq n}\left\|f_{k}\right\|_{L_{\varphi}}=\frac{e_{n}\left(L_{\varphi}\right)}{\varphi^{-1}(n / t)}
\end{gathered}
$$

and these in turn give, since $t>0$ was arbitrary, the desired left hand estimates. Since $L_{\varphi}=\left(L_{1}\right)_{\varphi}$ and $d_{n}\left(L_{1}\right)=1, e_{n}\left(L_{1}\right)=n$, so the required right hand estimates follow by Corollary 2.7 completing the proof. 


\section{The lattice COnstants \\ FOR MARCINKIEWICZ AND LORENTZ SPACES}

In this section we calculate the constants $e_{n}(M(\psi))$ and $d_{n}(\Lambda(\psi))$ for Marcinkiewicz and Lorentz spaces, respectively. We recall that if $(\Omega, \mu)$ is a measure space and $\psi:[0, \mu(\Omega)) \rightarrow[0, \infty)$ is an increasing concave function with $\psi(0)=0$, then the Lorentz space $\Lambda(\psi)$ is defined to be the r.i. space of all $f \in L^{0}(\mu)$ such that

$$
\|f\|_{\Lambda(\psi)}:=\int_{0}^{\mu(\Omega)} f^{*}(s) d \psi(s)<\infty .
$$

The Marcinkiewicz space $M(\psi)$ is defined to be the r.i. space of all $f \in L^{0}(\mu)$ such that

$$
\|f\|_{M(\psi)}:=\sup _{0<t<\mu(\Omega)} \frac{\int_{0}^{t} f^{*}(s) d s}{\psi(s)}<\infty .
$$

We are ready to prove the main result of this section. We note that an application for (i) was found in the paper [9].

Theorem 3.1. Let $(\Omega, \mu)$ be a nonatomic measure space and let $\psi:[0, \mu(\Omega)) \rightarrow$ $[0, \infty)$ be an increasing concave function. For each positive integer $n$, let $\varphi(n):=$ $\sup _{0<t<\mu(\Omega)} \frac{n \psi(t / n)}{\psi(t)}$. Then the following statements are true:

(i) For the Marcinkiewicz space $M(\psi)$ on $(\Omega, \mu)$ we have $e_{n}(M(\psi))=\varphi(n)$. In particular $e_{n}(M(\psi)) \asymp n \psi(1 / n)$ whenever $\psi$ is submultiplicative near 0 .

(ii) If $\psi(0+)=0$, and $\psi(t) \rightarrow \infty$ as $t \rightarrow \infty$ whenever $\mu(\Omega)=\infty$, then for the Lorentz space $\Lambda(\psi)$ on $(\Omega, \mu)$, we have $d_{n}(\Lambda(\psi))=\varphi(n)$. In particular $d_{n}(\Lambda(\psi)) \asymp n \psi(1 / n)$ whenever $\psi$ is submultiplicative near 0 .

Proof. (i). Fix $n \geq 2$ and suppose $f_{1}, \ldots, f_{n} \in M(\psi)$ are disjoint and nonnegative. Let $A_{1}, \ldots, A_{n} \in \Sigma$ be disjoint with $s_{k}=\mu\left(A_{k}\right)>0$ for $k=1, \ldots, n$. Let $A=$ $\bigcup_{k=1}^{n} A_{k}$. Since $\psi:[0, \mu(\Omega)) \rightarrow[0, \infty)$ is a concave function and

$$
\frac{1}{\psi\left(s_{k}\right)} \int_{A_{k}} f_{k} d \mu \leq\left\|x_{k}\right\|_{M(\psi)},
$$

we obtain for $f=f_{1}+\ldots+f_{n}$ and $s=s_{1}+\ldots+s_{n}$,

$$
\begin{aligned}
\frac{1}{\psi(s)} \int_{A} f d \mu & =\frac{1}{\psi(t)}\left(\sum_{k=1}^{n}\left(\frac{1}{\psi\left(s_{k}\right)} \int_{A_{k}} f_{k} d \mu\right) \psi\left(s_{k}\right)\right) \\
& \leq \frac{1}{\psi(s)}\left(\sum_{k=1}^{n} \psi\left(s_{k}\right)\right) \max _{1 \leq k \leq n}\left\|x_{k}\right\|_{M(\psi)} \leq \frac{n \psi(s / n)}{\psi(s)} \max _{1 \leq k \leq n}\left\|x_{k}\right\|_{M(\psi)} \\
& \leq \varphi(n) \max _{1 \leq k \leq n}\left\|x_{k}\right\|_{M(\psi)} .
\end{aligned}
$$

This implies that

$$
\left\|\sum_{k=1}^{n} f_{k}\right\|_{M(\psi)} \leq \varphi(n) \max _{1 \leq k \leq n}\left\|x_{k}\right\|_{M(\psi)}
$$

and whence $e_{n}(M(\psi)) \leq \varphi(n)$. To get the reverse inequality, fix $0<t<\mu(\Omega)$ and $n \geq 2$. Since $\mu$ is nonatomic measure, we may find a measurable subset $E \subset \Omega$ and disjoint measurable subsets $E_{1}, \ldots, E_{n}$ of $E$ with $\mu(E)=t, E=\bigcup_{k=1}^{n} E_{k}$ so that 
$\mu\left(E_{k}\right)=t / n$ for each $k=1, \ldots, n$. Then for $f_{k}=\chi_{E_{k}}, k=1, \ldots, n$, we have

$$
\begin{aligned}
\frac{t}{\psi(t)} & =\left\|\chi_{E}\right\|_{M(\psi)}=\left\|f_{1}+\ldots+f_{n}\right\|_{M(\psi)} \\
& \leq e_{n}(M(\psi)) \max _{1 \leq k \leq 1}\left\|f_{k}\right\|_{M(\psi)}=e_{n}(M(\psi)) \frac{t}{n \psi(t / n)} .
\end{aligned}
$$

Thus we conclude that for all $n$ and $0<t<\mu(\Omega)$,

$$
\frac{n \psi(t / n)}{\psi(t)} \leq e_{n}(M(\psi))
$$

This yields the desired formula. To conclude the proof of (i) it is enough to observe that $\psi$ being submultiplicative near 0 implies that $\varphi(n) \asymp n \psi(1 / n)$.

(ii). It is easy to check that our hypotheses on $\psi$ imply that $\Lambda(\psi)$ is ordercontinuous. Consequently $\Lambda(\psi)^{\prime}=M(\psi)$ with equality of norms (see [8]). Applying Proposition 2.3 we obtain that for each positive integer $n$ we have

$$
d_{n}(\Lambda(\psi))=e_{n}(M(\psi))=\varphi(n),
$$

and this completes the proof.

We show applications of the above theorem to the exponential Orlicz spaces $\operatorname{Exp} L^{\phi}:=L_{\Phi}$ on a finite measure space, where $\Phi(t)=\exp (\phi(t))-1$, and $\phi$ is an Orlicz function. It is well known that these spaces can also be described as Marcinkiewicz spaces. An easy proof when a measure space $(\Omega, \mu)$ is finite can be seen as follows. Note that the fundamental function of $\operatorname{Exp} L^{\phi}$ equals $\psi(t)=$ $1 / \phi^{-1}(\ln (1+1 / t))$ for $0<t \leq \mu(\Omega)$. Therefore $1 / \psi$ belongs to the Orlicz space $L_{\Phi}$ on $(0, \mu(\Omega))$ whenever $\mu$ is finite, from which it follows readily that $\operatorname{Exp} L^{\phi}=M(\psi)$, $\psi(t)=t \phi^{-1}(\ln (1+1 / t))$ for $t>0$. Likewise, it is also well known that the Lorentz $L(\log L)^{1 / \alpha}$ spaces can be described as Orlicz spaces (for more on this, see [2]).

Our next result is an extension of the Zygmund spaces $L(\log L)^{1 / \alpha}$, which is implicit in [7, Lemma 9.7] .

Corollary 3.2. Let $\phi$ be an Orlicz function, let $\psi(t) \asymp t \phi^{-1}(\ln (1+1 / t))$ for $t>0$, and let $(\Omega, \mu)$ be a finite nonatomic measure space. Then

$$
d_{n}(\Lambda(\psi)) \asymp \phi^{-1}(\ln (n+1)) .
$$

Proof. Since positive concave functions on $[0, \infty)$ are subadditive, $\phi^{-1}(u+v) \leq$ $\phi^{-1}(u)+\phi^{-1}(v)$ for every $u, v>0$. This implies that for every $0<t<\mu(\Omega)$ we have

$$
\phi^{-1}(\ln (1+n / t)) \leq \phi^{-1}(\ln (n+1))+\phi^{-1}(\ln (1+1 / t)),
$$

and whence (by $\mu(\Omega)<\infty$ )

$$
\varphi(n):=\sup _{0<t<\mu(\Omega)} \frac{n \psi(t / n)}{\psi(t)}=\sup _{0<t<\mu(\Omega)} \frac{\phi^{-1}(\ln (1+n / t))}{\phi^{-1}(\ln (1+1 / t))} \asymp \phi^{-1}(\ln (n+1)) .
$$

Thus the desired equivalence follows from Theorem 3.1 .

We remark that the generalized Orlicz space $\Lambda(\psi)_{\phi}$ generated by the Lorentz space $\Lambda(\psi)$ and an Orlicz function $\phi$ is equal to the Orlicz-Lorentz spaces $\Lambda_{\phi}(\psi)$. Then, combining Corollary 2.7 and Theorem 3.1. we obtain the estimate of the lattice constant $d_{n}\left(\Lambda_{\phi}(\psi)\right)$ for the Orlicz-Lorentz spaces $\Lambda_{\phi}(\psi)$. 


\section{ACKNOWLEDGMENT}

We thank Mario Milman for some useful comments about an earlier version of this paper.

\section{REFERENCES}

1. A. Aliprantis and O. Burkinshaw, Positive operators, Pure and Applied Mathematics Series No. 119, Academic Press, New York-London, 1985. MR809372 (87h:47086)

2. C. Bennett and R. Sharpley, Interpolation of operators, Academic Press, New York, 1988. MR 928802 (89e:46001)

3. Yu. A. Brudnyi and N. Ya. Krugljak, Interpolation Functors and Interpolation Spaces. I, North-Holland, Amsterdam, 1991. MR:1107298 (93b:46141)

4. A. V. Bukhvalov, Theorems on interpolation of sublinear operators in spaces with mixed norm, in: Qualititative and approximate methods for the investigation of operator equations, Yaroslav. Gos. Univ., Yaroslavl', 1984, 90-105 (in Russian). MR841773 (87h:46150)

5. A. P. Calderón, Intermediate spaces and interpolation, the complex method, Studia Math. 24 (1964), 113-190. MR0167830 (29:5097)

6. W. B. Johnson, B. Maurey, G. Schechtman and L. Tzafriri, Symmetric structures in Banach spaces, Mem. Amer. Math. Soc., Vol. 19, No. 217, 1979. MR527010 (82j:46025)

7. N. J. Kalton, Lattice Structures on Banach Spaces, Memoirs Amer. Math. Soc., Vol. 103, No. 493, 1993. MR1145663 (93j:46024)

8. S. G. Krein, Ju. I. Petunin and E. M. Semenov, Interpolation of linear operators. Translations of Mathematical Monographs, Vol. 54, American Mathematical Society, Providence, R.I., 1982. MR649411 (84j:46103)

9. T. Kühn and M. Mastyło, Weyl numbers and eigenvalues of abstract summing operators, J. Math. Anal. Appl. 369 (2010), no. 1, 408-422. MR.2643879 (2011c:47037)

10. J. Lindenstrauss and L. Tzafriri, Classical Banach spaces. II, Springer-Verlag, Berlin, 1979. MR.540367 (81c:46001)

11. G. Ya. Lozanovskii, Certain Banach lattices. IV, Sibirsk. Mat. Z. 14 (1973), 140-155 (Russian); English transl.: Siberian. Math. J. 14 (1973), 97-108. MR0336314 (49:1089)

12. G. Ya. Lozanovskii, Transformations of ideal Banach spaces by means of concave functions, in: Qualitative and approximate methods for the investigation of operator equations, Yaroslav. Gos. Univ., Yaroslavl' ${ }^{\prime}$ 1978, 122-147 (in Russian). MR559326 (83a:46042a)

13. V. I. Ovchinnikov, The methods of orbits in interpolation theory, Math. Rep. (Bucur.) 1 (1984), 349-516. MR877877 (88d:46136)

14. S. Reisner, On two theorems of Lozanovskii concerning intermediate Banach lattices, Geometrical Aspects of Functional Analysis-Israel Seminar 1986/87, Lecture Notes in Mathematics 1317, Springer-Verlag, Berlin-Heidelberg-New York-Tokyo, 1988, 67-83. MR950976 (89j:46019)

15. V. A. Shestakov, Transformations of Banach ideal spaces and the interpolation of linear operators, Bull. Polish Sci. Math. 29 (1981), 569-577 (Russian). MR654216 (83f:46030)

Faculty of Mathematics and Computer Science, Adam Mickiewicz University and Institute of Mathematics, Polish Academy of Science (Poznań branch), Umultowska 87, 61-614 Poznań, Poland

E-mail address: mastylo@amu.edu.pl 